\title{
A!
}

This is an electronic reprint of the original article.

This reprint may differ from the original in pagination and typographic detail.

Dubé, M.; Rost, M.; Elder, K.R.; Alava, M.; Majaniemi, S.; Ala-Nissila, T.

\section{Liquid conservation and nonlocal interface dynamics in imbition}

Published in:

Physical Review Letters

DOI:

10.1103/PhysRevLett.83.1628

Published: 01/01/1999

Document Version

Publisher's PDF, also known as Version of record

Please cite the original version:

Dubé, M., Rost, M., Elder, K. R., Alava, M., Majaniemi, S., \& Ala-Nissila, T. (1999). Liquid conservation and nonlocal interface dynamics in imbition. Physical Review Letters, 83(8), 1628-1631.

https://doi.org/10.1103/PhysRevLett.83.1628

This material is protected by copyright and other intellectual property rights, and duplication or sale of all or part of any of the repository collections is not permitted, except that material may be duplicated by you for your research use or educational purposes in electronic or print form. You must obtain permission for any other use. Electronic or print copies may not be offered, whether for sale or otherwise to anyone who is not an authorised user. 


\title{
Liquid Conservation and Nonlocal Interface Dynamics in Imbibition
}

\author{
M. Dubé, ${ }^{1,3, *}$ M. Rost, ${ }^{1,3, \dagger}$ K. R. Elder, ${ }^{2}$ M. Alava, ${ }^{3}$ S. Majaniemi,,${ }^{1,3}$ and T. Ala-Nissila ${ }^{1,3,4}$ \\ ${ }^{1}$ Helsinki Institute of Physics, P.O. Box 9, FIN-00014 University of Helsinki, Finland \\ ${ }^{2}$ Department of Physics, Oakland University, Rochester, Michigan 48309-4487 \\ ${ }^{3}$ Laboratory of Physics, P.O. Box 1100, FIN-02015 HUT, Espoo, Finland \\ ${ }^{4}$ Department of Physics, Brown University, Providence, Rhode Island 02912-1843
}

(Received 19 May 1999)

\begin{abstract}
The propagation and roughening of a liquid-gas interface moving through a disordered medium under the influence of capillary forces is considered. The system is described by a phase-field model with conserved dynamics and spatial disorder is introduced through a quenched random field. Liquid conservation leads to slowing down of the average interface position $H$ and imposes an intrinsic correlation length $\xi_{\mathrm{x}} \sim H^{1 / 2}$ on the spatial fluctuations of the interface. The interface is statistically self affine in space, with global roughness exponent $\chi \simeq 1.25$, and exhibits anomalous scaling.
\end{abstract}

PACS numbers: 68.35.Ct, 05.70.Ln, 47.55.Mh, 68.35.Fx

The dynamics of an invading liquid front in a disordered medium involves many problems of interest in statistical physics such as transport and diffusion in random media [1,2], roughening of a moving interface [3], and pinning [4] if the interface stops. Many experiments have been conducted on such phenomena [5], examples include Hele-Shaw cells [6] and the spontaneous imbibition of water in paper [7-11]. In the latter case, capillary forces drive the liquid until balanced by loss of water by evaporation or hydrostatic pressure. Often local theories such as directed percolation depinning [7,9] have been successful in describing the properties of such pinned fronts [4].

Local interface theories cannot, however, provide a complete description of imbibition since the transport of liquid to the front from the reservoir must be taken into account. In particular, this leads to a slowing down of the invading front even without evaporation or gravity [12]. In this Letter a model of imbibition that explicitly addresses this issue through the inclusion of liquid conservation is introduced. This is achieved by a generalized Cahn-Hilliard equation with a nonequilibrium boundary condition that couples the system to a liquid reservoir. Two different cases are considered: a liquid front invading a disordered medium without gravity or evaporation and a steady state front maintained at a constant distance from the liquid reservoir as done in the experiments of Horváth and Stanley [10].

An obvious consequence of liquid conservation is that the interface dynamics is nonlocal. One manifestation of this nonlocality is that the average interface height $H$ grows as $t^{1 / 2}$ as might be expected by Washburn's equation [12] (the velocity, $\dot{H} \sim 1 / H$ ). The interface is found to be superrough, with global roughness exponent $\chi \simeq 1.25$, and exhibits anomalous scaling [3,13]. Interface fluctuations saturate at a correlation length $\xi_{x}$, which is controlled by the average interface height such that $\xi_{\mathrm{x}} \sim H^{1 / 2}$. This implies that the average interface width $W \sim \xi_{x}^{X}$ [14]. The relationship between $\xi_{\mathrm{x}}$ and $H$ is a direct consequence of the conservation law and arises from the interplay between driving force and curvature. No such relation occurs in local models [5]. The temporal correlations of the interface qualitatively agree with the experiments of Ref. [10]. It is further found that the front moves by avalanches, as reflected in the behavior of the higher moments of the temporal correlations [15].

Phase-field model of imbibition. - To describe the liquid/gas system a locally conserved field $\phi(\mathbf{x}, t)$ is introduced such that $\phi=+1$ and -1 are the liquid and gas phases, respectively. The free energy functional of the system can then be written, $\mathcal{F}\{\phi\}=\int d \mathbf{x}\left[(\nabla \phi)^{2} /\right.$ $2+V(\phi)]$, where $V(\mathbf{x}, \phi) \equiv-\phi(\mathbf{x}, t)^{2} / 2+\phi(\mathbf{x}, t)^{4} /$ $4-\alpha(\mathbf{x}) \phi(\mathbf{x}, t)$. The liquid/gas interface is thus sustained in the standard manner by the gradient energy term and double well structure of $V$. The variable $\alpha(\mathbf{x})$ accounts for the random nature of the medium and has correlations; $\langle\alpha(\mathbf{x})\rangle=\bar{\alpha}, \quad$ and $\left\langle\alpha(\mathbf{x}) \alpha\left(\mathbf{x}^{\prime}\right)\right\rangle-\bar{\alpha}^{2}=$ $(\Delta \alpha)^{2} \delta\left(\mathbf{x}-\mathbf{x}^{\prime}\right)$. The propensity of the medium to absorb liquid can be controlled by $\alpha$ since it determines the local chemical potential $(\mu=-\delta F / \delta \phi)$. The dynamical evolution of $\phi$ is determined by a continuity equation $\partial_{t} \phi+\nabla \cdot \mathbf{j}=0$, where $\mathbf{j}(\mathbf{x}, t)=-\nabla \mu(\mathbf{x}, t)$. The resulting equation of motion, i.e.,

$$
\partial_{t} \phi=-\nabla^{2}\left[\nabla^{2} \phi+\phi-\phi^{3}+\alpha(\mathbf{x})\right],
$$

is a slightly modified Cahn-Hilliard equation [16]. The absorption of liquid by a dry random medium can be modeled with the appropriate boundary conditions. To reflect the presence of an infinite resevior at $y \leq 0$ the chemical potential at $y=0$ is fixed to be zero. To induce the propagation of the front, the top end of the system is kept "dry" [i.e., $\phi(y \rightarrow \infty)=-1$ ] and $\bar{\alpha}>0$. Equation (1) can be augmented to include evaporation by adding a term proportional to $(\phi+1)$ [17].

This model was specifically constructed to incorporate several essential physical features of imbibition. More precisely the model incorporates the conserved nature of the fluid which leads directly to a Gibbs-Thomson 
boundary condition on the liquid front and an average front velocity that decreases in time. To see these properties and gain more insight into the model it is useful to project out the equation of motion of the interface using standard projection operator methods $[18,19]$. Expanding to the lowest order in the interfacial curvature $(\mathcal{K})$ and $\bar{\alpha}$ [20] gives

$$
\Delta \phi \int_{-\infty}^{\infty} d x^{\prime} \mathcal{G}\left(x, h \mid x^{\prime}, h^{\prime}\right) \dot{h}^{\prime}=\eta(x, h)+\sigma \mathcal{K},
$$

where $G$ is the half-plane Green's function given by $\mathcal{G}\left(x, y \mid x^{\prime}, y^{\prime}\right)=\frac{1}{4 \pi} \ln \left(\frac{\left(x-x^{\prime}\right)^{2}+\left(y-y^{\prime}\right)^{2}}{\left(x-x^{\prime}\right)^{2}+\left(y+y^{\prime}\right)^{2}}\right) \quad$ which reflects a broken translational symmetry along the $y$ axis due to the reservoir. The interface is defined by the single-valued function $h(x, t), \sigma \equiv \int d y\left(\phi_{0}^{\prime}\right)^{2}$ is the surface tension, $\phi_{0}$ is the one-dimensional kink solution of Eq. (1), and $\Delta \phi=2$ is the miscibility gap. The quenched noise can be written $\eta(x, h) \equiv \int d y \phi_{0}^{\prime}[y-$ $h(x, t)] \alpha(x, y) \sim 2 \alpha(x, h)$ in the sharp interface limit. Similar nonlocal equations also arise in the context of Laplacian fluid flow [21] and step growth [22]. Previous theoretical work on equations for interfaces at the depinning transition [4] and nonlinear equations with long range kernels [23] does not apply to the situation encountered here.

The Gibbs-Thomson condition, $\left.\mu\right|_{\text {int }} \sim \mathcal{K}$ can be immediately obtained from Eq. (2) in the limit $\dot{h}=0$ since $\eta$ is the chemical potential at the interface. In addition since $\phi$ is a conserved field it is easy to show that the normal interface velocity is proportional to the gradient of $\mu$ at the interface; i.e., $\Delta \phi v_{n}=-\left.\partial_{n} \mu\right|_{\text {int }}$. Further information can be obtained by linearizing in $h$ to obtain

$$
\dot{h_{k}}\left(1-e^{-2|k| H}\right)+|k| \dot{H} h_{k}\left(1+e^{-2|k| H}\right)=|k|\left(\eta_{k}-\sigma k^{2} h_{k}\right),
$$

where $h_{k}$ are the Fourier components of $h$ and $H=h_{0}$ is the average interface position [24]. Equation (3) can now be used to obtain an expression for the average interface position (Washburn's equation) and lateral correlation length as follows.

Washburn's equation. - In the limit $k \rightarrow 0$, Eq. (3) reduces to $\dot{H}=\bar{\alpha} /(2 H)$. Thus, a planar interface, without evaporation, is never pinned and moves as $H(t)=$ $(\bar{\alpha} t)^{1 / 2}$. This is a standard result known as Washburn's equation [12] and can be understood as follows: In the absence of a liquid reservoir $\mu=-\bar{\alpha}$ throughout the system. When a reservoir is included at the lower boundary $\mu$ is set to zero at $y=0$. For a slowly moving front $\mu(y)$ satisfies a diffusion equation in the bulk phases with the quasistationary solution $\mu \approx-\bar{\alpha} y / H$ for $y \leq H$ and $\mu(y)=-\bar{\alpha}$ for $y \geq H$. This result is equivalent to Washburn's equation since $\dot{H}=v_{n}=-\left(\left.\partial_{n} \mu\right|_{\text {int }}\right) / \Delta \phi$. In essence, the gradient in $\mu$ creates a current from the reservoir towards the interface, causing it to advance.

Lateral correlation length.-Another useful result coming from Eq. (3) is the existence of a lateral length scale separating two different modes of damping of the interface fluctuations. Long wavelength fluctuations are damped by the advancing motion of the front (i.e., $|k| \dot{H} h_{k}$ ), while short wavelength fluctuations are damped by surface tension (i.e., $\sigma|k|^{3} h_{k}$ ). The length scale separating these two modes $\xi_{\mathrm{x}} \sim(\sigma / \dot{H})^{1 / 2}=(\sigma H /$ $\bar{\alpha})^{1 / 2}$ is closely related to the Mullins-Sekerka instability of driven Laplacian fronts [25], although the situation is reversed here. Because fluid is transported towards the front from behind, advanced (retarded) parts receive less (more) mass than the average and the front is stabilized at long length scales.

This result can be understood in more physical terms as follows: Because of the Gibbs-Thomson effect a local "bulge" of vertical extent $W$ and lateral size $\xi$ alters the chemical potential by $\Delta \mu \simeq \sigma W / \xi^{2}$. On the other hand, the average gradient in $\mu$ in the bulk liquid induces a difference $\Delta \mu \simeq \bar{\alpha} W / H$ across a vertical distance $W$. These two differences balance each other at a length given by

$$
\xi_{\mathrm{x}} \simeq \sqrt{\sigma H / \bar{\alpha}} .
$$

By this mechanism, one expects the front to be smoother on length scales larger than $\xi_{\mathrm{x}}$ as compared to smaller scales. While these interface results provide useful insight into the front propagation the behavior was examined in more detail by direct numerical simulation.

Numerical integrations. - Equation (1) was integrated on a square grid with spacing $\Delta x=1$ using various lateral system sizes $L_{x}$. The disorder $\alpha(\mathbf{x})$ was a random variable on each grid point. Different distributions (exponential, Gaussian, etc.) gave the same results. The interface position $h(x, t)$ was determined by a linear interpolation of the zero of the phase field, $\phi[x, h(x, t)]=0$, with overhangs (hardly present here) cut off.

Figure 1 shows the rise of an initially flat front. Fronts are shown at equal time intervals displaying Washburn behavior $H^{2}(t)=\bar{\alpha} t$, also shown in Fig. 1. The vertical and lateral extent of interface fluctuations increase with height. The early time behavior of the width $W(t)=$ $\left\langle\overline{[h(x, t)-\bar{h}]^{2}}\right\rangle^{1 / 2}$, where the overbar denotes a system average and the brackets denote the average over the disorder, is also shown in Fig. 1. It is found that $W(t) \sim$ $t^{\beta}$ with $\beta=0.32 \pm 0.02$.

In a recent experiment of paper wetting [10], a steady state situation was reached by pulling the paper sheet at a constant velocity $\mathbf{v}=-v \hat{\mathbf{y}}$ towards the reservoir. To model this case, Eq. (1) can be written as

$$
\begin{aligned}
\partial_{t} \phi= & -\nabla^{2}\left[\nabla^{2} \phi+\phi-\phi^{3}+\alpha(\mathbf{x}-\mathbf{v} t)\right] \\
& +\mathbf{v} \cdot \nabla \phi,
\end{aligned}
$$




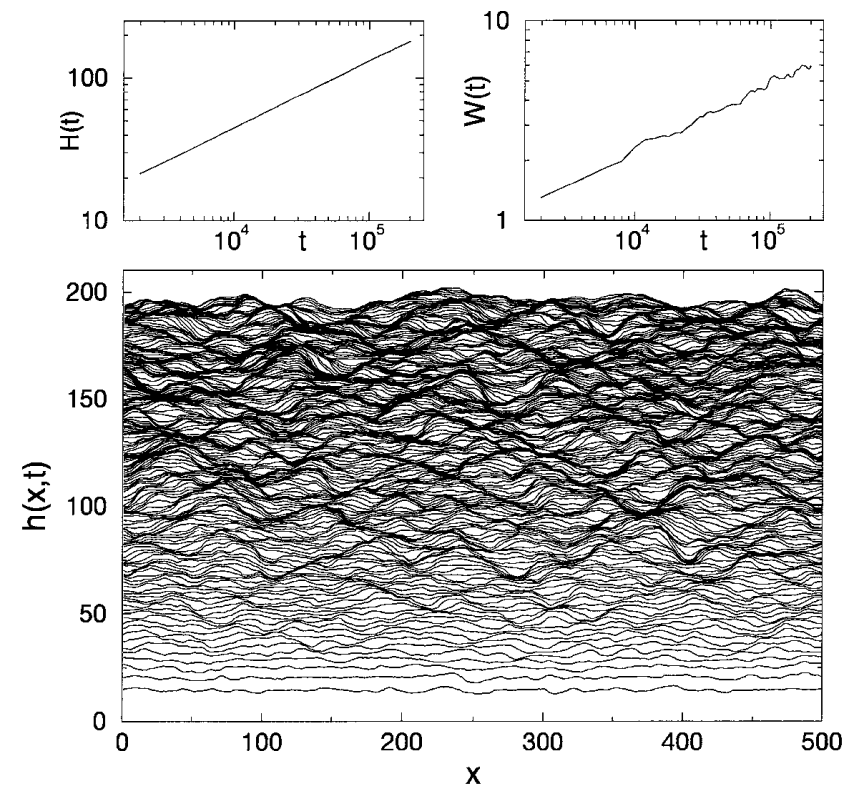

FIG. 1. Configurations of the rising interface at time intervals $\Delta t=10^{3}$. For this system, the average height $H \sim t^{0.49}$ and the width $W(t) \sim t^{0.32}$, according to least-square fits.

keeping the original boundary conditions. In this case, the interface remains at a fixed average height $H=\bar{\alpha} / 2 v$, at which a freely rising interface would have velocity $v$.

Equation (5) was also integrated numerically. The role of $\xi_{x}$ as a maximal range of correlated roughness becomes apparent in the steady-state structure factor $S(k, H)=\left\langle\overline{|h(k, t)|^{2}}\right\rangle$ and spatial height difference correlation function $G_{2}(r, H)=\left\langle\overline{[h(x+r, t)-h(x, t)]^{2}}\right\rangle^{1 / 2}$ [26], both shown in Fig. 2. The lateral fluctuations of the interface are correlated only up to $\xi_{\mathrm{x}}<L$. The global roughness exponent $\chi$ is taken from the power law decay of $S\left(k>\xi_{\mathrm{x}}^{-1}, H\right) \sim k^{-(2 \chi+1)}=k^{-3.5 \pm 0.2}, \quad$ yielding $\chi \simeq 1.25$. The relation $\xi_{\mathrm{x}} \sim v^{-1 / 2} \sim(H / \bar{\alpha})^{1 / 2}$ is confirmed in Fig. 2, were the spatial correlation function is rescaled as

$$
G_{2}(r, H)=\Delta \alpha v^{-\chi / 2} g\left(r v^{1 / 2}\right),
$$

for different $v$ (i.e., different $H$ ) and $\Delta \alpha$. The scaling function $g(u)$ is constant for $u \gg 1$ and $g(u) \sim u^{\chi_{\text {loc }}}$ if $u \ll 1$, with $\chi_{\text {loc }} \simeq 1$. The interface is thus superrough, and $G_{2}(r, H)$ shows anomalous scaling in the sense that $\chi_{\text {loc }}<\chi . G_{2}(r, H)$ increases with $H$ for all $r[3,13]$.

Quasistationary rise. - The numerical data indicate that spatial correlations in the interface position are the same for both sets of simulations. This can be seen in Fig. 2 where steady state correlation functions at fixed heights $H$ are compared with the correlation functions of a freely rising front, obtained from Eq. (1), at times $t_{H}=H^{2} / \bar{\alpha}$. This implies that the interface is always in a quasistationary state in the freely rising case. The scaling form, Eq. (6), is thus also valid for the freely rising front provided that the time dependence $H(t)=(\bar{\alpha} t)^{1 / 2}$ is used, such that $\xi_{\mathrm{x}} \sim(t / \bar{\alpha})^{1 / 4}$ [19]. This indicates that
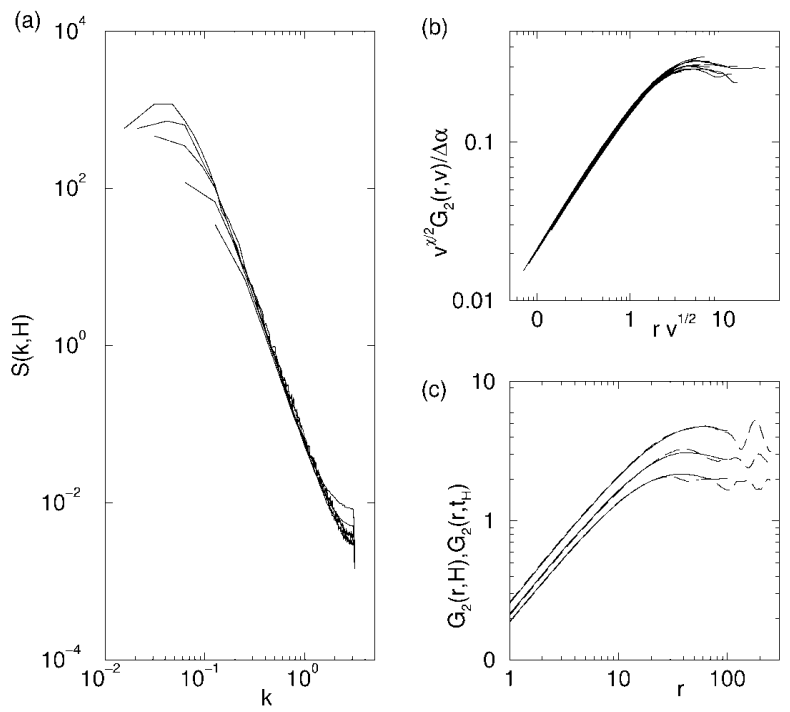

FIG. 2. (a) Structure factors for systems of height $H=50$, 100,150 , and 200 and $L_{x}=2 H$. (b) Correlation functions $G_{2}(r, H)$ scaled according to Eq. (6) for various $v \sim H^{-1 / 2}$ and $\Delta \alpha$. (c) Comparison of the correlation functions in the steady state, $G_{2}(r, H)$ (solid lines) for $H=25,50$, and 100 and of the rising case $G_{2}\left(r, t_{H}\right)$ (dashed lines), at times $t_{H}=H^{2} / \bar{\alpha}$.

the dynamical correlation length $\xi_{t} \sim t^{1 / z}$ increases at a rate faster or equal to $t^{1 / 4}$ so that the interface fluctuations can always catch up with the maximal correlation range. This also implies $W \sim \xi_{x}^{\chi} \sim t^{\chi / 4}=t^{\beta}$, with $\beta \simeq 0.31$, in agreement with $W(t)$ shown in Fig. 1.

Temporal correlations. - To compare with the experimental work of Ref. [10], the temporal correlations in the steady state, obtained from Eq. (5), were analyzed through the function $C_{q}(t, H)=\left\langle\overline{|h(x, t+s)-h(x, s)|^{q}}\right\rangle^{1 / q}$ also taking higher moments $q>2$ into account. The early time logarithmic slopes of the higher moments ( $\beta_{q}$ ) decrease with $q$, as shown in the inset of Fig. 3, in contrast to the spatial $G_{q}$ for which $\chi_{q}$ is independent of $q$. This lack of scaling reflects the propagation of the front by avalanches (easily visible in Fig. 1), also found for local interfaces at the depinning transition $[15,19]$.

Nevertheless, our model agrees with the experiments in three ways, visible in the main part of Fig. 3: (i) the $\beta_{q}$ are independent of $H$; (ii) the late time saturation level of $C_{q}$ increases with $H$ indicating larger overall roughness; and (iii) $C_{q}(t, H)$ at fixed small $t$ decreases with $H$, indicating faster intrinsic avalanche velocity for smaller $H$. Quantitatively our result $\beta_{2}=0.85 \pm 0.04$ differs from the experimental value 0.56 but so does the average interface velocity $v \sim H^{-1.6}$ in Ref. [10] compared to the Washburn behavior in our model [27]. Since water flow dynamics in real paper can be complicated by various phenomena such as, e.g., fiber swelling [19], the disagreement is not too surprising and should be clarified in future investigations.

Conclusion. - We have constructed a simple but flexible model for spontaneous imbibition of a liquid from 


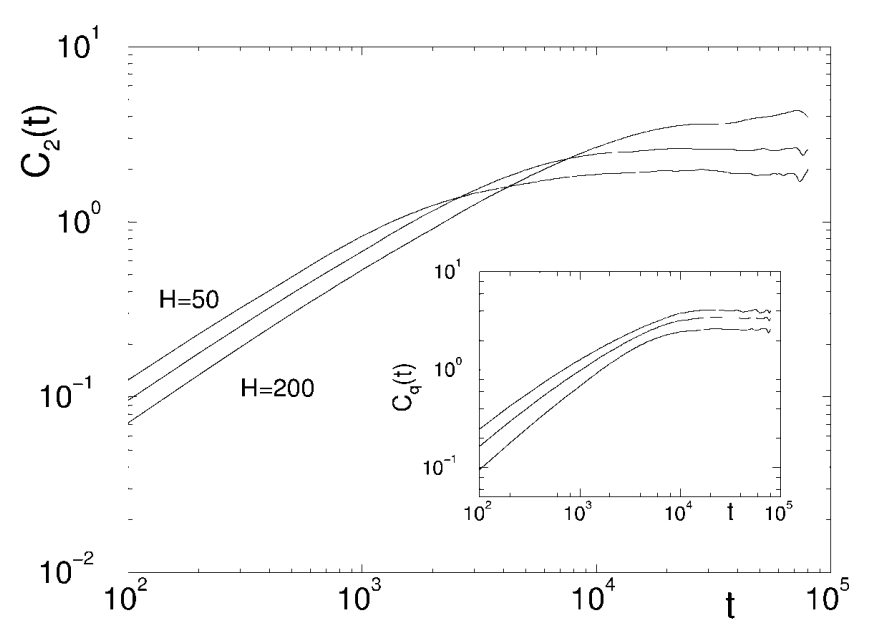

FIG. 3. Steady state temporal correlation functions for systems of size $L_{x}=400$, at heights $H=50,100$, and 200. In the inset, the moments $q=2,4$, and 6 (from bottom to top) for $H=100$ are compared. $C_{2}$ has exponent $\beta_{2}=0.85$ while, for the higher moments, the effective exponents $\beta_{4} \approx 0.76$ and $\beta_{6} \approx 0.69$.

a reservoir into a disordered medium. Conservation of liquid leads to Washburn's relation for the average height $H \sim t^{1 / 2}$ [12], a behavior which does not occur naturally in local interface models. The interface dynamics becomes nonlocal, and an intrinsic length scale $\xi_{x}$ emerges as given by Eq. (4). This length scale is a distinct prediction of the model and intimately relates the fluctuations of the interface with its average progression. The existence and dynamical increase of this region should be experimentally easier to observe than precise values of scaling exponents on scales $r<\xi_{\mathrm{x}}$. From the model, spatial scaling is found to be anomalous with a global roughness exponent $\chi \simeq 1.25$, similar to what has been seen in other models of driven interfaces in disordered media [28]. The growth exponent $\beta$ is explained in terms of $\xi_{\mathrm{x}}$ and $\chi$.

This work has, in part, been supported by the Academy of Finland and a Research Corporation Grant No. CC4787 (KRE).

*Corresponding author.

Electronic address: dube@pcu.helsinki.fi

${ }^{\dagger}$ Corresponding author.

Electronic address: rost@pcu.helsinki.fi

[1] J.P. Bouchaud and A. Georges, Phys. Rep. 195, 127 (1990).

[2] A. E. Scheidegger, The Physics of Flow Through Porous Media (MacMillan Co., New York, 1957).

[3] J. Krug, Adv. Phys. 46, 139 (1997).

[4] M. Kardar, Phys. Rep. 301, 85 (1998).

[5] A.-L. Barabási and H.E. Stanley, Fractal Concepts in Surface Growth (Cambridge University Press, Cambridge, England, 1995).
[6] M. A. Rubio, Phys. Rev. Lett. 63, 1695 (1989); V. K. Horváth, F. Family, and T. Vicsek, J. Phys. A 24, L25 (1991); S. He, G. L. M. K. S. Kahanda, and P.-z. Wong, Phys. Rev. Lett. 69, 3731 (1992); T. Delker, D. B. Pengra, and P.-z. Wong, Phys. Rev. Lett. 76, 2902 (1996); A. Dougherty and N. Carle, Phys. Rev. E 58, 2889 (1998).

[7] S. V. Buldyrev et al., Phys. Rev. A 45, R8313 (1992).

[8] F. Family, K. C. B. Chan, and J. G. Amar, in Surface Disordering: Growth, Roughening and Phase Transitions, edited by R. Jullien, J. Kertész, P. Meakin, and D. E. Wolf (Nova Science, Commack, 1992).

[9] L. A. N. Amaral et al., Phys. Rev. Lett. 72, 641 (1994).

[10] V. K. Horváth and H. E. Stanley, Phys. Rev. E 52, 5166 (1995).

[11] O. Zik et al., Europhys. Lett. 38, 509 (1997).

[12] E. W. Washburn, Phys. Rev. 17, 273 (1921). See also O. Zik et al., Phys. Rev. E 58, 689 (1998).

[13] J. M. López, M. A. Rodríguez, and R. Cuerno, Phys. Rev. E 56, 3993 (1997).

[14] We only consider the case of small amplitudes in interface fluctuations. In the presence of substantial overhangs one expects $W \sim \xi_{\mathrm{x}}$.

[15] Z. Olami, I. Procaccia, and R. Zeitak, Phys. Rev. E 49, 1232 (1994); H. Leschhorn and L. H. Tang, ibid. 49, 1238 (1994).

[16] J. W. Cahn and J.E. Hilliard, J. Chem. Phys. 28, 258 (1958).

[17] Hydrodynamical effects could also be included, following Ref. [18]. However, in some applications, such as water imbibition in paper, we do not expect long range hydrodynamical flow since it is broken by the fibers. In such a case, the field $\alpha(\mathbf{x})$ represents a coarse-grained capillary pressure.

[18] K. Kawasaki and T. Ohta, Prog. Theor. Phys. 68, 129 (1982); D. Jasnow and J. Viñals, Phys. Fluids 7, 747 (1996).

[19] M. Dubé et al. (to be published).

[20] The expansion is around a stationary planar interface. In such an expansion the small parameters are the dimensionless quantities, $\zeta \mathcal{K}$ and $\zeta \bar{\alpha} / H$, where $\zeta$ is the interfacial thickness.

[21] J. Krug and P. Meakin, Phys. Rev. Lett. 66, 703 (1991).

[22] G.S. Bales and A. Zangwill, Phys. Rev. B 41, 5500 (1990); W. K. Burton, N. Cabrera, and F. C. Frank, Philos. Trans. R. Soc. London A 243, 299 (1951).

[23] S. Mukherji and S. M. Bhattacharjee, Phys. Rev. Lett. 79, 2502 (1997).

[24] Equation (3) acquires a local form in the limit $|k| H \ll 1$. Two points on the interface separated by a distance larger than $H$ are decoupled.

[25] W. W. Mullins and R. F. Sekerka, J. Appl. Phys. 35, 444 (1964).

[26] The argument $H$ instead of $t$ indicates a steady state, at the average height $H$.

[27] $\beta_{2}$ is not the exponent $\beta$ of the early time increase of the rms width in the freely rising case, contrary to the suggestion made in Ref. [10], since the latter is determined through $\xi_{\mathrm{x}}$.

[28] H. Leschhorn, Physica (Amsterdam) 195A, 324 (1993); S. Roux and A. Hansen, J. Phys. I (France) 4, 515 (1994). 